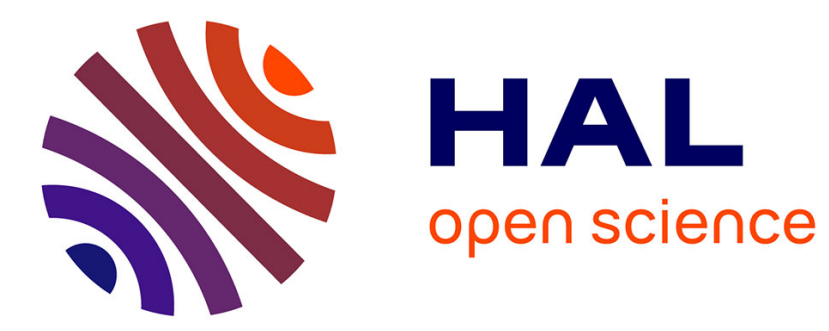

\title{
Les places des adolescent.e.s en zone urbaine sensible, entre attribution, appropriation et retrait
}

\author{
Isabelle Danic
}

\section{To cite this version:}

Isabelle Danic. Les places des adolescent.e.s en zone urbaine sensible, entre attribution, appropriation et retrait . Les Annales de la Recherche Urbaine, 2016, 111. hal-01429479

\section{HAL Id: hal-01429479 \\ https://hal.science/hal-01429479}

Submitted on 31 Aug 2018

HAL is a multi-disciplinary open access archive for the deposit and dissemination of scientific research documents, whether they are published or not. The documents may come from teaching and research institutions in France or abroad, or from public or private research centers.
L'archive ouverte pluridisciplinaire HAL, est destinée au dépôt et à la diffusion de documents scientifiques de niveau recherche, publiés ou non, émanant des établissements d'enseignement et de recherche français ou étrangers, des laboratoires publics ou privés. 


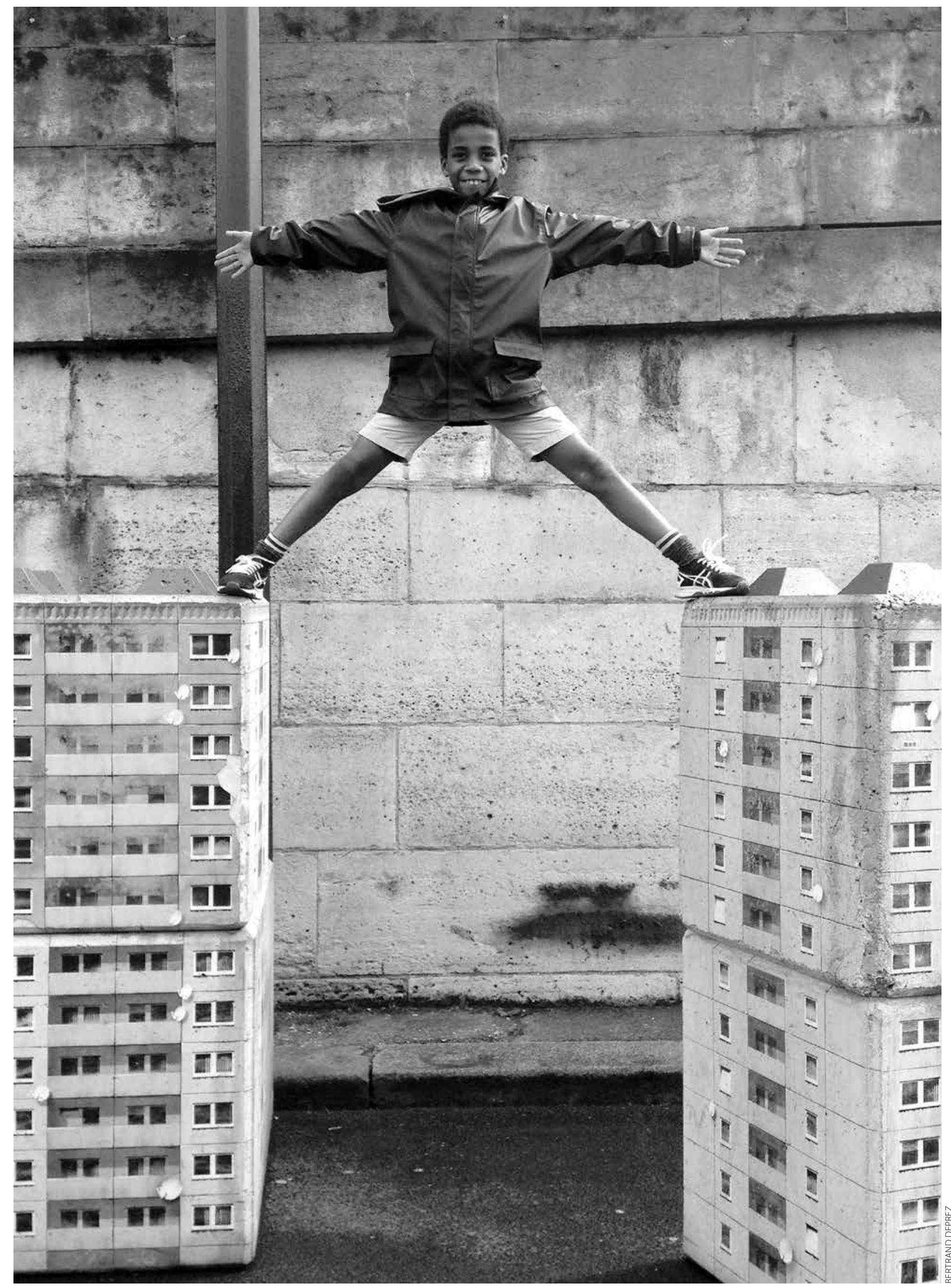




\section{Les places des adolescents.e.s en zone urbaine sensible,} entre attribution, appropriation et retrait

\section{Isabelle Danic}

Cet article vise à cerner la place des adolescentes et des adolescents dans les zones urbaines sensibles (Zus) ${ }^{1}$, et la façon dont elle s'est constituée, tout en cherchant à savoir si les adolescent.e.s se l'approprient telle quelle ou s'ils la redéfinissent. Bien que constituant un cinquième de la population française, les moins de seize ans ont longtemps été négligés par les sciences sociales francophones. C'est à partir des années 1990 que l'adolescence, entendue comme âge social produit en grande part par la scolarisation au collège (Danic, Delalande et Rayou, 2006 ; Danic, 2006), commence à être examinée sous ses aspects scolaire (Dubet et Martucelli, 1996 ; Zaffran, 2000), sanitaire (Choquet, 2005), culturel (Bruno, 2000 ; Octobre, 2004 ; Octobre et al., 2010), ou de façon plus globale, dans ses dimensions sociale, familiale et scolaire (Lepoutre, 1997 ; Rubi, 2005 ; Singly de, 2006). Ces travaux font apparaître les pratiques et les représentations adolescentes de l'espace urbain dans la mesure où $60 \%$ des adolescents vivent en ville 2 . Cependant, la question de l'occupation de l'espace urbain par les adolescents n'est posée explicitement que dans les années $2000^{3}$ : sur la scène politique lors des campagnes électorales avec les mesures visant à réguler l'usage de l'espace public par les adolescents ; sur la scène médiatique avec les émeutes

1. Les zones urbaines sensibles sont des quartiers catégorisés ainsi par décrets ; elles sont définies dans la loi de novembre 1996 comme " caractérisées [notamment] par la présence de grands ensembles ou de quartiers d'habitat dégradé et par un déséquilibre accentué entre l'habitat et l'emploi». On en dénombre 751. Elles sont définies par l'Insee comme des « territoires infra-urbains définis par les pouvoirs publics pour être la cible prioritaires de la politique de la ville, en fonction des considérations locales liées aux difficultés que connaissent les habitants de ces territoires ".

2. $26 \%$ des adolescents de 15 à 17 ans vivent en centre-ville et $34 \%$ en banlieue, $23 \%$ en secteur périurbain et $17 \%$ en milieu rural (Insee, 2006), soit $60 \%$ dans des pôles urbains.

3. Voir le travail précurseur de Kokoreff (1993). de 2005 ; et dans les sciences sociales, avec des analyses de ces évènements (Beaud et Pialloux, 2005 ; Mauger, 2006 ; Lagrange et Oberti, 2006 ; Le Goaziou et Mucchielli, 2006) ; puis des travaux sur l'inscription ordinaire des adolescents dans l'espace public (Kebabza, 2004 ; Ménard et Zucker, 2007 ; Bréviglieri, 2007 ; Bréviglieri et Cicchelli, 2007 ; Zaffran, 2005 ; Faure, 2008 ; Oppenchaim, 2011)4. Ces études montrent que, tendanciellement, les adolescents garçons de milieu populaire ${ }^{5}$ se retrouvent plus souvent entre eux et « dehors ", alors que les adolescentes de milieu populaire et les adolescent.e.s des classes moyennes et supérieures restent davantage à leur domicile, stationnent peu à l'extérieur et fréquentent davantage les institutions culturelles. En étudiant les mobilités d'adolescent.e. $s^{6}$ de Zus en Île-de-France, N. Oppenchaim (2011) montre qu'ils pratiquent davantage d'activités à proximité de leur domicile, telles que la promenade et les visites amicales, et ce du fait des moindres possibilités parentales (en termes économique, temporel et de moyens de transport), comparé aux autres adolescents franciliens, qui pratiquent davantage d'activités de loisirs structurées et de visites familiales. De plus, il apparaît que cette moindre disponibilité des familles vivant en Zus restreint les investissements extérieurs des adolescentes, que les parents veulent

4. Espace public est entendu dans son double sens d'espace de libre
circulation qui ne relève pas de l'appropriation individuelle, régi par
un droit de visite et un droit de regard et au sens imagé de lieu de la
citoyenneté ordinaire confrontant différents acteurs.

5. Dans ces travaux, les classes populaires renvoient aux catégories des ouvriers et des employés.

6. L'enquête d'Oppenchaim porte sur des jeunes de quinze à dixhuit ans résidant dans différentes communes de la petite couronne parisienne.

Les Annales de la recherche urbaine $n^{\circ} 111,2016$, pp. 78-89, (C)MEDDE, Puca. 
davantage surveiller à cet âge, sans pouvoir les accompagner en permanence.

À partir de données collectées auprès d'adolescents de quatorze et quinze ans ${ }^{7}$, cet article tente de préciser la place des adolescents filles et garçons, et surtout la constitution de cette place dans l'espace des deux Zus investiguées. La notion de marquage semble appropriée pour étudier cette inscription spatiale des acteurs sociaux dans ses deux versants, subi (la place affectée aux adolescent.e.s) et actif (la place que les adolescent.e.s se définissent) (première partie). Elle permet une approche originale, qui présente la place construite pour les adolescent.e.s par les politiques publiques, redéfinie par la mise en œuvre des professionnels et par les habitants (seconde partie). En contrepoint, elle révèle la place que les adolescentes et les adolescents se constituent par leur propre marquage (fréquentation ou non des équipements, appropriation de certains espaces publics) et comment cela se différencie entre eux (troisième partie).

\section{La notion de marquage comme outil d'analyse de la construction de la place}

Selon Brunet et al. dans leur Dictionnaire critique de géographie (1992), « la place est un emplacement, une portion d'espace occupée par un objet, une personne [...]. Par extension et abstraitement, [...] la place est définie par sa position et définit une situation, y compris au sens figuré de ces mots ». Pour appréhender la place des adolescents garçons et filles dans leur quartier et éclairer la dynamique qui la définit et la redéfinit en permanence, la notion de marquage semble heuristique ${ }^{8}$. Veschambre (2006) a défini le marquage comme "une production de marques dans un espace dont l'appropriation est affirmée ou revendiquée » ou un "réinvestissement de traces », trace étant entendue comme « ce qui subsiste du passé $[. .$.$] ou ce qui est considéré comme tel » (Ripoll,$ 2006) : les constructions, les édifices, les sculptures, les monuments, le mobilier urbain, les inscriptions, les graffitis, les plantations. On peut y ajouter les façons de s'habiller, de s'exprimer, de se tenir, à la suite de Pinçon et Pinçon-Charlot (1989; 2007). Le marquage peut être visuel (présence, mobilité, activité), sonore (bruit, parole), olfactif (odeurs naturelles ou artificielles). Pour Tersigni et Bonny (2010), le marquage peut être conçu « au sens actif et passif de marquer la différenciation et d'en être marqué » :

7. La longueur de l'article ne permet ni une comparaison des deux Zus, au demeurant peu différentes en la matière, ni une présentation des changements observés au fil de nos terrains. D'autres publications en feront état.

8. Cet article se nourrit notamment du séminaire « Marques, marqueurs et traces. Le marquage des différences dans l'espace » d'Eso-Rennes, mené en 2008 sous la responsabilité de Yves Bonny et Simona Tersigni. le marquage peut être actif (par exemple la construction d'un équipement destiné aux adolescents, ou une prise de parole dans l'espace public), ou passif (le fait d'être mal habillé dans un quartier chic par exemple). La production de marques est à la fois concrète et symbolique puisqu'elle a toujours une signification, qu'il s'agisse d'appartenance, d'appropriation, de revendication, ou d'attribution, voire de valorisation ou de stigmatisation (appropriation des espaces valorisés par les populations aisées, attribution des espaces stigmatisés aux démunis). L’enjeu du marquage de l'espace n'est donc pas seulement spatial mais d'abord social, voire politique : il peut viser à marquer sa présence, son existence, affirmer un droit de présence, revendiquer une reconnaissance sociale.

Investiguer la place des filles et garçons adolescents dans une Zus en utilisant la notion de marquage amène d'une part à traiter les adolescents comme des sujets marqués et marquants l'espace urbain, et d'autre part à cerner le marquage dont ils sont l'objet par l'action et les significations d'autrui (décideurs politiques, professionnels, habitants), mais aussi leur propre marquage, qu'ils réalisent en tant qu'acteurs, certes dominés, mais disposant cependant de ressources.

\section{Méthodologie}

L'analyse porte sur des matériaux collectés lors de deux enquêtes en zones urbaines sensibles (Zus) d'une ville moyenne, Rennes (210 000 habitants), I'une menée dans la Zus Villejean en 2006, 2007, et 2010, et l'autre dans la Zus Maurepas en 2013 et 2014. Elles n'ont pas été conçues ensemble au départ, mais sont rapprochées rétrospectivement. Construites dans les années 1960, les deux Zus de Villejean (13000 habitants) et de Maurepas (9000 habitants) ${ }^{9}$ se caractérisent par leur concentration d'habitat social et une proportion notable d'habitants en difficulté socioéconomique le revenu médian des ménages à Villejean est de 1028 euros, celui de Maurepas est de 950 euros contre 1600 euros pour la ville entière. Dans les deux quartiers, la population est aussi plus jeune que dans la ville ( $22 \%$ de moins de 17 ans pour les cinq territoires prioritaires rennais contre $16 \%$ pour ville entière), et plus mixte (15\% de population immigrée dans les cinq territoires prioritaires rennais contre $7,5 \%$ dans la ville entière $\left.{ }^{10}\right)$. Le quartier de Villejean a été réaménagé dans les années 2000 avec l'arrivée du métro en 2002. Le quartier de Maurepas connaît actuellement un réaménagement autour de sa future station de métro, en lien avec la construction de la deuxième ligne prévue pour 2018.

À Villejean, le corpus est constitué d'entretiens individuels avec 34 adolescents, 8 professionnels, un entretien collectif avec 7 jeunes, des parcours commentés avec prise de vue avec 9 adolescents, un questionnaire auprès de 60 collégiens. À Maurepas, le corpus est constitué d'entretiens individuels avec 15 adolescents, 4 professionnels (chargé de mission Politique de la ville, animateurs, directeur d'équipement), un entretien collectif avec 14 adolescents. En outre, des observations ont été réalisées dans les deux quartiers, que viennent compléter des documents papier ou numériques (sites Internet) issus des organismes et des structures concernés, ainsi que deux monographies réalisées dans le cadre de l'ANR Juvenil (David et al., 2009 ; 2010).

9. À Rennes, les cinq territoires prioritaires rassemblent $25 \%$ de la population rennaise.

10. Données Insee 2009. 


\section{Le marquage dont les adolescent.e.s sont l'objet}

Les politiques publiques préstructurent les places des adolescent.e.s dans les Zus enquêtées, et leurs mises en œuvre par les professionnels, les normes habitantes et les encadrements familiaux contribuent à définir des places distinctes dans le quartier pour les adolescents filles et garçons.

\section{Une place octroyée par les politiques publiques}

Les politiques de la ville, les politiques familiales et scolaires, les politiques de la jeunesse, les politiques sociales et sanitaires, la politique de sécurité, définies aux échelles nationale, régionale et locale, participent fortement à définir la place de ces adolescents. D'abord, à l'échelle nationale, les lois et les normes assignent l'adolescence à l'espace familial, pour son entretien et son éducation, et à l'espace scolaire pour son instruction. En vis-à-vis, l'espace public n'est pas considéré comme pleinement légitime pour les jeunes (Ménard et Zucker, 2008). Il s'agit donc de créer d'autres espaces pour l'adolescence, notamment par la politique de la ville, dont les jeunes constituent la cible préférée (Loncle, 2007) au travers de ses dispositifs ${ }^{11}$ : création de lieux d'accueil, d'activités de loisir, de sports, espaces de prévention. Parallèlement, durant les années 2000, des politiques publiques se sont développées visant à réguler l'usage de l'espace public par les adolescents : interdiction des regroupements dans les halls d'immeuble, pénalisation des incivilités et des désordres publics, lutte contre l'alcool et la toxicomanie, éducation à la citoyenneté.

À l'échelle locale, tant la Région Bretagne, le Département d'Ille-et-Vilaine que la Ville de Rennes se préoccupent depuis longtemps du social et des jeunes, que ce soit sous les majorités de droite - avec une approche « catholique sociale »-, ou sous les majorités de gauche ${ }^{12}$ - avec une tradition de partenariat entre le public et le milieu associatif. Sur les sites d'enquête, l'acteur majeur de l'intervention publique vis-à-vis des adolescents, après l'État (avec la politique scolaire et la politique de la ville), est la Ville de Rennes, dont la politique envers les adolescents se caractérise par la pérennité des orientations et des acteurs. Depuis les années 1960, la prise en charge des adolescents est organisée selon un

11. Dans les deux quartiers enquêtés, les adolescents sont concernés par la Mission locale, l'atelier Santé Ville, le contrat local sécurité, la gestion urbaine de proximité, le projet de réussite éducative, le plan local pluriannuel pour l'insertion et l'emploi.

12. La majorité était de droite jusqu'en 2004 pour la région et le département, jusqu'en 1977 pour la ville de Rennes. Actuellement, la région, le département d'Ille-et-Vilaine et la ville de Rennes sont dirigés par le parti socialiste, ce qui facilite l'accord sur des orientations communes. maillage des quartiers du territoire municipal, s'appuyant sur la délégation aux associations spécialisées ou aux associations socioculturelles généralistes. Présentes dans les deux Zus enquêtées, les associations spécialisées dans l'encadrement des adolescents sont anciennes : le cercle Paul Bert et l'Union des Patros rennais datent de la fin XIX siècle, auxquels se sont ajoutés depuis les années 1960 Le Relais (prévention spécialisée), les maisons des jeunes et de la culture et les maisons de quartier (qui n'interviennent pas seulement auprès des adolescents). Si l'encadrement des adolescents est délégué aux associations, la Ville en conserve le pilotage au travers de sa politique de subventions pour créer une prise en charge compensatrice d'un milieu familial et social supposé démuni. Dans le même objectif, la Ville de Rennes a également monté des partenariats actifs avec les bailleurs sociaux - notamment une politique d'animation de proximité par la gestion conjointe des locaux collectifs de l'habitat social, et avec la Caisse d'allocations familiales (Caf) d'Ille-et-Vilaine ${ }^{13}$. La mise en place des politiques de la ville (développement social des quartiers en 1989, puis contrat de ville) s'est appuyée sur les acteurs socio-éducatifs " historiques ", en introduisant cependant une formalisation et une systématisation d'actions collectives antérieures, et des fonds complémentaires. Dans le cadre des contrats de ville, le cercle Paul Bert, l'Union des Patros rennais et Le Relais sont amenés à collaborer dans différents quartiers rennais, avec le souci d'impliquer les jeunes et de les faire participer aux diverses expérimentations menées. Actuellement, le réseau d'acteurs locaux continue de travailler en complémentarité, en valorisant le consensus. En termes de financement, c'est la Ville de Rennes qui prend en charge les équipements ainsi que les salaires des personnels, avec le soutien de l'État et du conseil régional.

Fruits de ces politiques (Augustin, 2001), divers lieux municipaux sont affectés aux adolescents dans les deux quartiers Zus : outre les collèges publics, des terrains de sport, une bibliothèque, une maison de quartier (Villejean) ou une maison des associations (Maurepas), des équipements socio-éducatifs gérés par le cercle Paul Bert et par d'autres associations $^{14}$, avec des activités sportives, culturelles, un accompagnement scolaire et un accueil informel.

13. Cf. O. David (2009; 2010). Outre leur mission de prestations légales, les Caf ont une seconde mission de mise en œuvre d'une politique d'action sociale appropriée à son territoire $(5 \%$ du budget, soit 44 millions d'euros en 2007 en Ille-et-Vilaine). La jeunesse est une priorité de la Caf d'Ille-et-Vilaine, mise en œuvre par l'accompagnement des collectivités locales, dont la Ville de Rennes, au travers des contrats enfance-jeunesse et de la cogestion des centres sociaux, de partenariat avec des associations ou des institutions inscrite dans l'action sociale (ex. cercle Paul Bert).

14. Outre le cercle Paul Bert, on trouve à Villejean la Maison verte dédiée aux 10-20 ans qui propose des activités et un accueil informel, et à Maurepas, les Cadets de Bretagne, rattaché à l'Union des Patros, qui offrent diverses activités sportives. 
L'affectation de ces espaces aux adolescents est explicitée par leurs noms ( club ado », « espace jeunes »), et également par l'apparence des bâtiments (tags sur la Maison verte à Villejean et sur l'Espace jeunes du CPB à Maurepas).

Sur ce marquage concret de leur place dans l'espace public urbain se superpose un marquage symbolique par les discours des acteurs concernés - de la mairie, de la communauté de commune, de la préfecture, de la police, de la Justice, de l'Éducation nationale. Dans un contexte national stigmatisant les « jeunes de banlieue » perçus comme menace de délinquance et de criminalité, la vision des adolescents des quartiers à Rennes est plutôt celle de victimes d'un contexte urbain défavorable qu'il faut compenser par l'action publique. Selon Loncle (David et al., 2009 ; 2010), cette représentation de l'adolescent du quartier sensible s'est même améliorée, avec en vis-à-vis la dégradation de la figure de l'étudiant.e. La représentation de l'étudiant fêtard transgressif, née avec les nouvelles pratiques festives au centre-ville en 2004-2005 ${ }^{15}$, a localement revalorisé les adolescents des quartiers sensibles et légitimé les efforts pour leur donner une place, des lieux et des activités dans leurs quartiers. Au final, dans les deux quartiers investigués, une place est offerte aux adolescents, filles et garçons, par les équipements financés par une municipalité soucieuse de l'encadrement de la jeunesse des Zus.

\section{Une place redéfinie par la mise en œuvre des professionnels}

En deçà des intentions politiques, la place des adolescents dans l'espace des zones urbaines sensibles se concrétise par l'action des professionnels engagés sur le quartier. Dans les différents équipements enquêtés dans les deux quartiers, les professionnels rencontrés proposent des accueils informels et des activités plus structurées ; ils sont actifs pour impliquer les adolescents. Ce faisant, ils ajoutent au cantonnement scolaire une séparation extrascolaire des adolescents dans les équipements socio-éducatifs du quartier. Cependant, ils les incitent aussi à formuler des " projets ", des souhaits en matière d'activités, hors de la structure, hors du quartier voire de la ville, développant ainsi une exploration de nouveaux lieux.

15. Rennes accueille environ 60000 étudiants dans les différents établissements d'enseignement supérieur. En 2004-2005, face à l'occupation nocturne de l'espace public de l'hypercentre par des petits groupes bruyants et consommant de l'alcool, la préfète de région adopte des mesures répressives : fermeture anticipée des bars, interdiction de consommation dans l'espace public, intervention de la force publique pour disperser les jeunes «stagnant » dans l'hypercentre au-delà d'une heure. Les réponses des services déconcentrés de l'État et des acteurs locaux ont visés à encadrer les pratiques festives et à développer des actions de santé publique - ce que l'on ne présentera pas ici puisque ces actions visent le public étudiant.
Dans l'ensemble, une différenciation entre garçons et filles s'opère, les équipements socio-éducatifs y contribuent en accueillant beaucoup plus de garçons, y compris lors des accueils encadrés par des animatrices. Cet état de fait recoupe ce qui a été observé dans d'autres zones urbaines. Mais l'explication par les politiques publiques qui viseraient à canaliser la violence des garçons (Raibaud, 2007) est insuffisante ici. Si elle est juste à l'échelle nationale pour la politique de la ville, elle l'est beaucoup moins dans la situation locale enquêtée. À Rennes, dès les années 1990, l'Association pour la promotion et la recherche en action sociale (Apras) a fait remonter le constat d'une faible présence des adolescentes dans les équipements socio-éducatifs lors de plusieurs études réalisées pour la Ville. Depuis, cette dernière incite les structures à développer la mixitél ${ }^{16}$. Toutefois, cet objectif semble difficile à atteindre, et tous les professionnels ne reprennent pas à leur compte cette injonction. Plusieurs animateurs ou animatrices rencontrés disent leurs lieux ouverts tant aux unes qu'aux autres, admettent la moindre fréquentation des adolescentes et tentent de s'adapter en leur proposant des activités particulières. Les sorties proposées aux seules adolescentes ont du succès (sortie à l'Aquatonic ; mini-séjour à Paris), mais l'accueil réservé aux adolescentes du vendredi en fin d'après-midi dans l'une des structures n'a pas eu la fréquentation escomptée. Au-delà des « bonnes intentions » politiques, les professionnel.le.s sont assez démuni.e.s pour intervenir vis-à-vis des rapports sociaux de sexe, auxquels ils ne sont ni sensibilisés ni formés. La directive municipale de prendre en charge de façon similaire filles et garçons apparaît à nombre d'animateurs et animatrices « de première ligne » comme un discours politiquement correct déconnecté de la réalité de terrain. Les structures s'emploient à montrer la présence de filles dans leurs bilans d'activité pour obtenir les financements municipaux dont ils ont besoin pour fonctionner, sans pour autant envisager de modifier les relations sociales locales, qu'ils acceptent souvent sous couvert de relativisme culturel.

Manifestement, les places des adolescents et des adolescentes ne sont pas définies par les politiques publiques à elles seules, d'autant moins que les politiques nationales et locales peuvent diverger. Ces directives sont traduites par les professionnel.le.s qui se les approprient dans leurs propres perspectives, souvent sans formation sur la question des rapports de genre. De fait, une offre d'équipements et d'activités indifférenciée pour les filles et les garçons ne

16. Organisme regroupant la Ville, la Caf et les bailleurs sociaux, l'Association pour la promotion et la recherche en action sociale (Apras) a contribué à transformer l'encadrement des adolescents dans la ville, notamment par des recherches-actions qui ont diffusé auprès des élus, des institutions et des associations un point de vue commun valorisant notamment la mixité, et plus largement les jeunes et leur parole (David et al., 2009 ; 2010). 


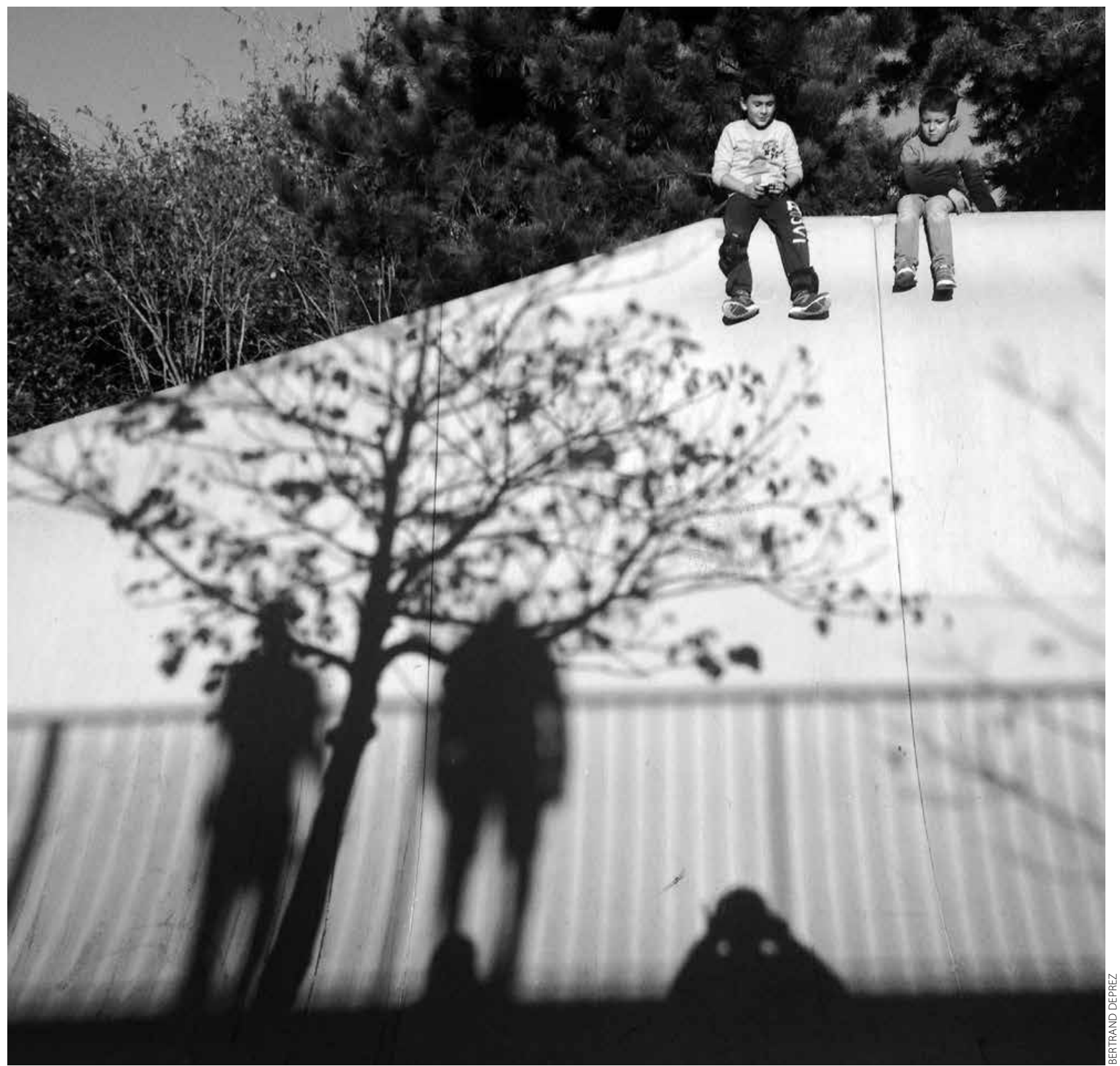

produit pas une appropriation indifférenciée. Les places des garçons et des filles se constituent également en articulation avec les normes d'occupation des espaces publics des habitants et avec les types d'encadrement parental.

\section{Un marquage d'appropriation des espaces publics par des habitants}

Les normes du quartier et les encadrements familiaux construisent une place pour les garçons dans les espaces publics, qui sont surtout occupés par des groupes d'adolescents garçons, et très peu par des adolescentes. La norme d'appropriation masculine des espaces publics est rapportée par les adolescentes (Danic, 2011) : «Chez nous, les filles, elles doivent pas rester dehors » (Samira). Et ces témoignages sont corroborés par nos observations : des hommes, jeunes et moins jeunes, signifient leur appropriation des lieux publics du quartier par un marquage fait de stationnement, d'interactions familières, par des propos ou des gestes dévalorisants, voire menaçants (Callu et al., 2005 ;Zegnani, 2013) : « [Sur la dalle commerçante], y a beaucoup de jeunes, ils te regardent bizarrement, c'est surtout qu'ils sont en bande et ils te regardent bizarrement quand tu passes. C'est embêtant de passer devant eux » selon Fadia ${ }^{17}$. En suivant Welzer-Lang (2002), le marquage masculin d'appropriation des espaces publics pourrait constituer une «stratégie de défense collective en réponse à la peur du chômage, à l'état de non-droit, à la souffrance de ne pouvoir exhiber d'autres attributs que la virilité ».

17. Tous les prénoms sont fictifs. 


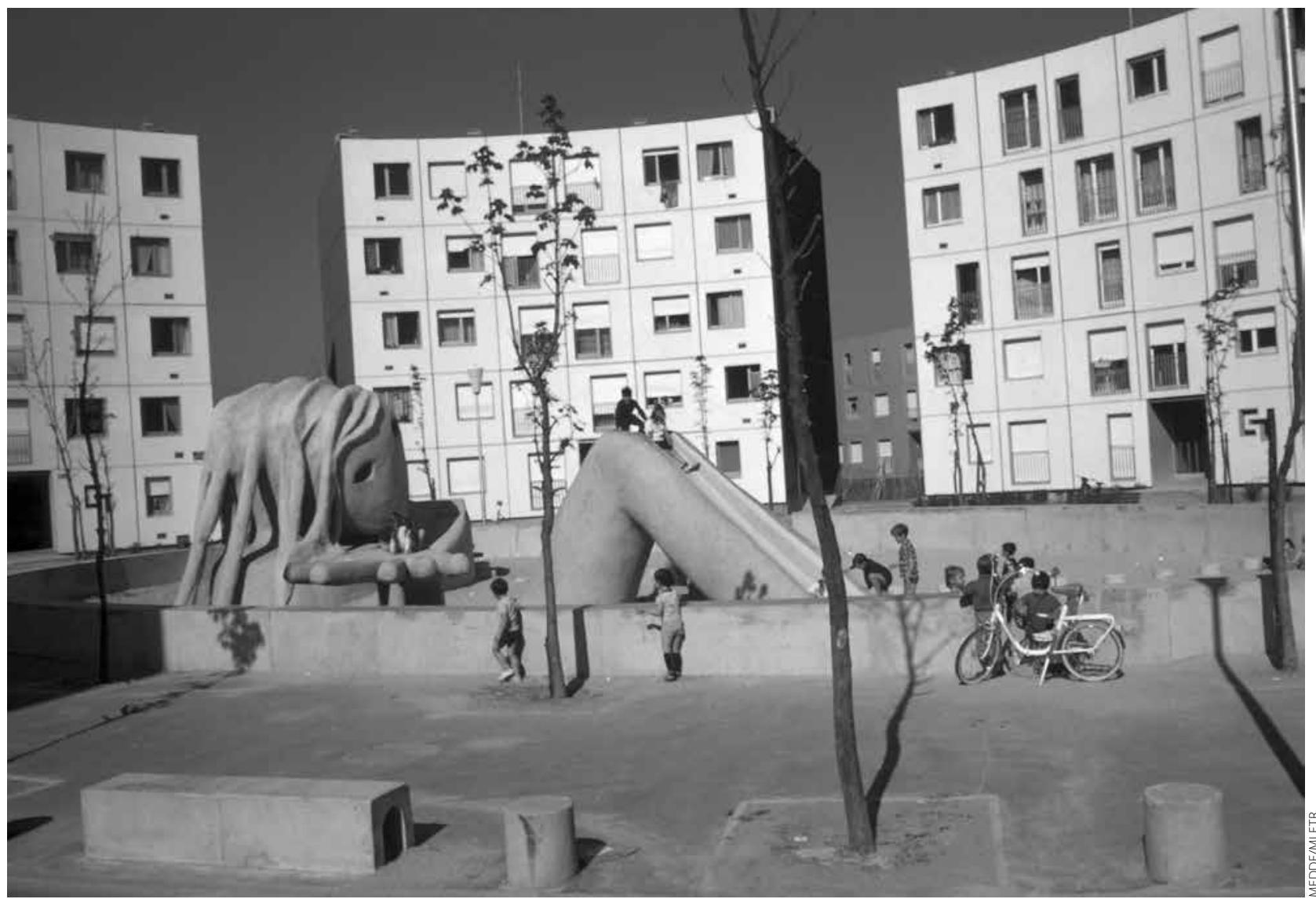

Ces normes locales d'appropriation masculine des lieux publics sont reprises par une partie des adolescents et légitimées en tant que reconstruction culturelle (« la place des hommes, des femmes, chez "nous" " pour les jeunes de parents maghrébins, africains, turcs) et/ ou reconstruction historique ("la place des hommes, des femmes avant »). En vis-à-vis, les adolescentes ont le sentiment de ne pas avoir de place dans l'espace public du quartier et de ne pouvoir l'occuper qu'en y « passant », pour les déplacements domicile-collège, les courses, ou les visites à la famille.

L'encadrement familial participe aussi à la construction de la place des adolescents et des adolescentes dans le quartier. Dans les deux Zus, on peut distinguer schématiquementdeuxidéaux-types defamilles: desfamilles résidentes en retrait du quartier, et qui soustraient leurs adolescent.e.s à l'environnement proche par la dissuasion ou par l'interdiction, mais autorisent leur sortie au centreville $^{18}$; et des familles qui s'identifient et participent à la vie du quartier, autorisent leurs adolescent.e.s à sortir dans les environs, à fréquenter les équipements, mais dissuadent ou interdisent les sorties au centre-ville. Dans les deux types, les parents limitent davantage l'investissement extérieur des filles parce qu'ils considèrent que les filles sont plus

18. Ces adolescents ont été rencontrés au collège. vulnérables que les garçons et/ou qu'elles doivent avoir de bonnes raisons pour sortir. À la question "Qu'est ce qui t'empêche d'aller dans certains endroits ou de passer par certains itinéraires? », les trois quarts des filles répondent soit "mes parents », et ce trois fois plus souvent que les garçons, soit les «personnes inquiétantes » (deux fois plus souvent que les garçons), soit « les jeunes », puis « le manque de transport ». Plus de la moitié des garçons ne répondent pas à cette question, manifestant ainsi qu'elle ne se pose pas pour eux ; ceux qui le font mentionnent " autre ", puis « mes parents ", « les personnes inquiétantes » et le «manque de transport ». Il apparaît que les familles résidant dans la Zus ne définissent pas les mêmes places dans le quartier pour leurs adolescents selon leur rapport au quartier et selon le sexe de l'adolescent ${ }^{19}$. Dans les familles qui s'identifient au quartier, les adolescent.e.s peuvent sortir dans le quartier, même si les filles doivent avoir des finalités précises pour le faire (visites, courses, activités encadrées). Pour les parents en retrait, il y a une limitation des investissements dans la Zus, plus forte encore pour les filles que pour les garçons.

Ces constats dans deux zones urbaines sensibles de Rennes convergent avec ceux réalisés dans des zones urbaines sensibles toulousaine (Kebabza,

19. Également selon l'âge, mais nous avons enquêté auprès d'adolescents de 14-15 ans. 
2004), stéphanoise (Faure, 2008), ou franciliennes (Oppenchaim, 2011). À Rennes, les politiques publiques locales ont pour objectif explicite de donner une même place aux adolescents filles et garçons. Cet objectif, qui se traduit par la création de lieux socio-éducatifs destinés aux adolescents des deux sexes et des financements conditionnés à une fréquentation mixte, est difficilement traduit en mesures concrètes par les professionnels, démunis face au marquage genré du quartier par une partie des habitants. En conséquence, lorsqu'ils sont autorisés à investir le quartier par leurs parents, les adolescents garçons peuvent y trouver aisément une place, au contraire des filles, que ce soit aux terrains de foot, dans les rues ou dans les équipements socio-éducatifs.

\section{Le marquage de leur place par les adolescents et les adolescentes}

Selon son insertion familiale et sociale dans le quartier, chaque adolescent.e du quartier perçoit "sa " place, celle qui lui est proposée, dévolue par l'environnement proche. Pour autant, cette place ne devient pas sienne mécaniquement : il ou elle se l'approprie, ou la conteste, la modifie, l'élargit. Les principaux lieux assignés que sont le domicile et le collège font l'objet d'une appropriation affective et identitaire par les adolescents rencontrés qui développent un attachement à leur logement, en tant que lieu de la vie familiale, et au collège en tant que lieu de sociabilité amicale. S'ils acceptent dans leur grande majorité ces deux places ${ }^{20}$, ils en redéfinissent l'occupation pour y inclure des activités de sociabilité entre pairs ${ }^{21}$.

À côté des places assignées que sont leur collège et leur logement, la place des adolescent.e.s dans leur quartier est celle qu'ils et elles prennent dans les structures socioéducatives et dans l'espace public. En matière de fréquentation des équipements, les adolescents des Zus enquêtés pratiquent globalement moins d'activités encadrées que les adolescents d'autres quartiers, et en particulier les filles ${ }^{22}$. Quelques dizaines d'adolescent.e.s fréquentent les activités sportives proposées au sein des collèges par l'Union nationale du sport scolaire le mercredi après-midi, ou les activités proposées à un coût modique par les équipements socio-éducatifs de leur quartier (clubs sportifs, patinoire, cinéma, et plus exceptionnellement paint ball, karting...). Quelques dizaines d'adolescents garçons et des filles, jusqu'à environ

20. Nous ne traiterons pas dans cet article le cas des adolescents en rupture familiale et/ou scolaire, qui se définissent une autre place.

21. Pour le rapport au collège, voir Dubet et Martucelli (1996), et Zaffran (2000). Concernant l'espace familial, voir Singly de (2006).

22. Comparaison des données quantitatives et qualitatives collectées auprès des collégiens issus de la Zus et des secteurs environnants. Ces constats convergent avec ceux d'Oppenchaim en Zus francilienne. treize ans, notamment ceux qui participent à des activités encadrées, fréquentent les accueils informels offerts par plusieurs structures. Quelques dizaines d'adolescents filles et garçons participent aux ateliers d'accompagnement scolaire proposés dans les deux quartiers. Au final, sachant qu'environ 2860 personnes de moins de dix-sept ans résident à Villejean et 1980 à Maurepas, on constate qu'une grande part des adolescents ne fréquentent pas les équipements, les uns du fait d'un interdit parental (cas des familles en retrait du quartier), les autres par retrait délibéré. Ces derniers sont souvent des filles à partir de treize ans et de certains garçons, qui ne s'y sentent pas à leur place. À l'accueil informel d'un équipement socioéducatif, l'une des deux filles présentes (celle-ci a seize ans et l'autre onze) au milieu d'une dizaine d'adolescents garçons m'explique : "Les filles ne viennent pas parce qu'elles ont peur. Peur des gars, des grands. De toute façon, y a moins de filles que de gars dans le quartier. »

À côté des lieux dédiés qu'ils s'approprient diversement selon le rapport familial au quartier et selon leur genre, d'autres espaces publics urbains peuvent être revendiqués par les adolescent.e.s selon le marquage qu'ils y opèrent. Mais il reste la place que les adolescent.e.s se font de leur propre initiative dans la zone urbaine sensible, sans qu'elle leur soit attribuée.

\section{Marquer sa place par le corps}

Dans les espaces publics des deux quartiers, le marquage par les adolescents est le fait d'une minorité d'entre eux. Pour autant, elle est manifeste et remarquable. Hors des temps familiaux et scolaires, en stationnant ou en déambulant collectivement dans divers espaces du quartier, ces adolescents se différencient fortement au plan visuel (présence, mouvement, démarche, activités, look), sonore (parole, musique, bruits, usage de pétards) pour s'accaparer un emplacement, ou manifester leur existence. Au quotidien, le marquage mobilise largement le corps par des façons particulières de marcher, de se tenir (allure de cow-boy des garçons par exemple), de s'habiller, de se coiffer. Tout un ajustement se réalise sciemment pour se signaler comme adolescent « vrai » garçon, sûr de soi et dynamique : vêtement sportif et baskets, cheveux courts, casquettes. Cette visibilité est amplifiée par la parole : formes d'expression (ton vif, niveau sonore), langage avec un vocabulaire et une syntaxe particuliers - emprunt à des langues des populations immigrées, à l'argot, à la langue rom $^{23}$. À travers ce marquage, ces adolescents se définissent une identité d'âge distincte de l'enfance et de l'âge adulte,

23. De façon exceptionnelle, le marquage prend une forme spectaculaire par des actes violents vis-à-vis des biens (casser des vitres ou détruire du mobilier d'une structure) ou des personnes (« taper des vieux »), $c f$. Danic, 2011. 
et une identité de genre. Cette signification identitaire croise une signification d'appropriation : il s'agit aussi d'affirmer un «droit de présence » (Ripoll, 2006), ou plus fondamentalement un droit de cité (Danic, 2011), un droit d'existence mis à mal par une situation sociale et scolaire défavorable (Beaud et Pialloux, 2002 ; 2005)

En conséquence, le marquage des adolescents dans l'espace du quartier permet une différenciation externe, à l'égard d'autres catégories d'âge, et une différenciation interne au sein des adolescents. La place des adolescents dans les espaces publics des deux Zus est celle que certains, issus de familles qui s'identifient et s'impliquent dans le quartier, s'approprient. En vis-à-vis, les adolescents des familles en retrait ou d'autres quartiers doivent se faire discrets, ne pas marquer ce quartier, sous peine d'être considérés comme provocateurs. Certaines " manières d'habiter » des adolescents de quinze à dix-huit ans des Zus franciliennes étudiés par N. Oppenchaim se retrouvent chez les adolescents de quatorze et quinze ans des deux Zus rennaises : les " adolescents du quartier », des garçons qui occupent l'espace du quartier sans beaucoup en sortir ; les « filles de bonnes familles » qui occupent le quartier avec discrétion ; les « encadrés » qui sont plus souvent des filles, mais pas exclusivement, dont les parents considèrent qu'ils ne doivent pas sortir dans la Zus ; des "flâneurs ", qui occupent alternativement le quartier et des espaces urbains anonymes (plus souvent garçons en Île-de-France, plus souvent des filles à Rennes); et des "flâneurs exclusifs », qui sont plutôt des filles, qui rejettent le quartier et sortent au centreville. En revanche, à Rennes, nous n'avons pas rencontré de "guerrières ", bien que certains adolescents nous aient évoqué quelques filles "qui se prennent pour des garçons », ni de «crapuleuses » telles que les décrit Rubi (2005), ou de " passionnés » (Oppenchaim, 2011) qui désinvestissent le quartier au profit d'une activité-passion. Ces absences peuvent tenir à la moindre ampleur de l'investigation rennaise et à l'écart d'âge de la population étudiée, voire à des caractéristiques locales.

\section{La place des filles et la place des garçons}

Le marquage des adolescents dans les espaces publics visibles et valorisés est davantage le fait des adolescent.e.s «forts » au sens du quartier ${ }^{24}$ : force physique, force sociale (nombre d'alliés familiaux ou amicaux), force verbale (avoir de la répartie, savoir vanner, se marrer). Ce sont plus souvent des garçons ou des filles qui ont un allié masculin connu dans le quartier (frère, père...). Proche de la « fille de bonne famille » de la typologie d'Oppenchaim, Karima

24. Un adolescent « fort » dans le quartier peut être un adolescent « faible », au sens du statut social, de reconnaissance dans d'autres espaces (collège, centre ville...), cf. Danic, 2011. circule dans le quartier et fréquente le cercle Paul Bert (le soutien scolaire et la salle de danse). Adolescente disposant d'alliés masculins (père, oncle) dans le quartier, elle a aussi une stratégie pour ne pas être importunée dans l'espace public : "Faut pas qu'une fille, elle a l'air gentil et tout ça. Faut qu'elles soient méchantes avec leur regard, qu'elles sont des filles fortes. Dans la rue, mes copines me disent "t'as l'air méchante". Dans la rue, j’ai mes yeux fâchés. » Dans les lieux publics les plus valorisés localement, tels que la dalle commerciale, le commerce de kebab, l'équipement socio-éducatif, les terrains de sport, les adolescents « forts » stationnent aisément, adoptent des postures, des gestes, des attitudes très assurés de " propriétaires ", et éloignent ceux qui leur paraissent des intrus par le verbe (moquerie, insulte) ou par le geste (cracher au passage de quelqu'un, menace).

Face aux consignes parentales et à l'appropriation virile des lieux publics du quartier, la plupart des jeunes filles et une partie des jeunes garçons ${ }^{25}$ investissent davantage leurs domiciles, évitent ces lieux appropriés par les ados « forts » ou ne font qu'y passer, ne stationnent pas dans l'espace public et visitent les équipements socioculturels pour des activités précises. Lors de leur déplacement dans le quartier, la plupart des adolescentes tentent de passer inaperçues en adoptant une attitude discrète et une tenue respectable, en évitant notamment de valoriser leurs atouts physiques : "Faut pas trop se montrer pour pas mettre les garçons mal à l'aise. Faut pas montrer sa poitrine par exemple » répond Sorina, qui porte d'ailleurs une écharpe cachant ses formes. La majorité des filles enquêtées respecte cette attente sociale de ne pas marquer l'espace public, sans adhérer nécessairement à leur affectation à la sphère privée : elles s'en distancient dans leur discours et en fréquentant des lieux du quartier moins visibles ou des secteurs hors quartier tels que le centreville, voire en se créant des lieux propres dans le quartier. Ainsi à Maurepas, l'Espace jeunes du cercle Paul Bert ouvert aux adolescents de onze à dix-sept ans le mercredi et le samedi après-midi, reçoit principalement des garçons. Aux mêmes moments, de façon informelle, une deuxième salle destinée à la danse est occupée par les adolescentes. Il semble que cet usage se soit instauré à leur initiative : elles demandent la clé de la salle pour y danser. Elles y dansent certes parfois, mais surtout s'y retrouvent. Ainsi, elles ont laissé le lieu officiel aux garçons mais ont créé officieusement un autre espace collectif pour elles.

25. La place des garçons du quartier qui n'adhèrent pas à la définition virile de l'homme supposerait une investigation spécifique : ils sont encore plus difficiles à atteindre que les filles du quartier pour l'enquêteur. 


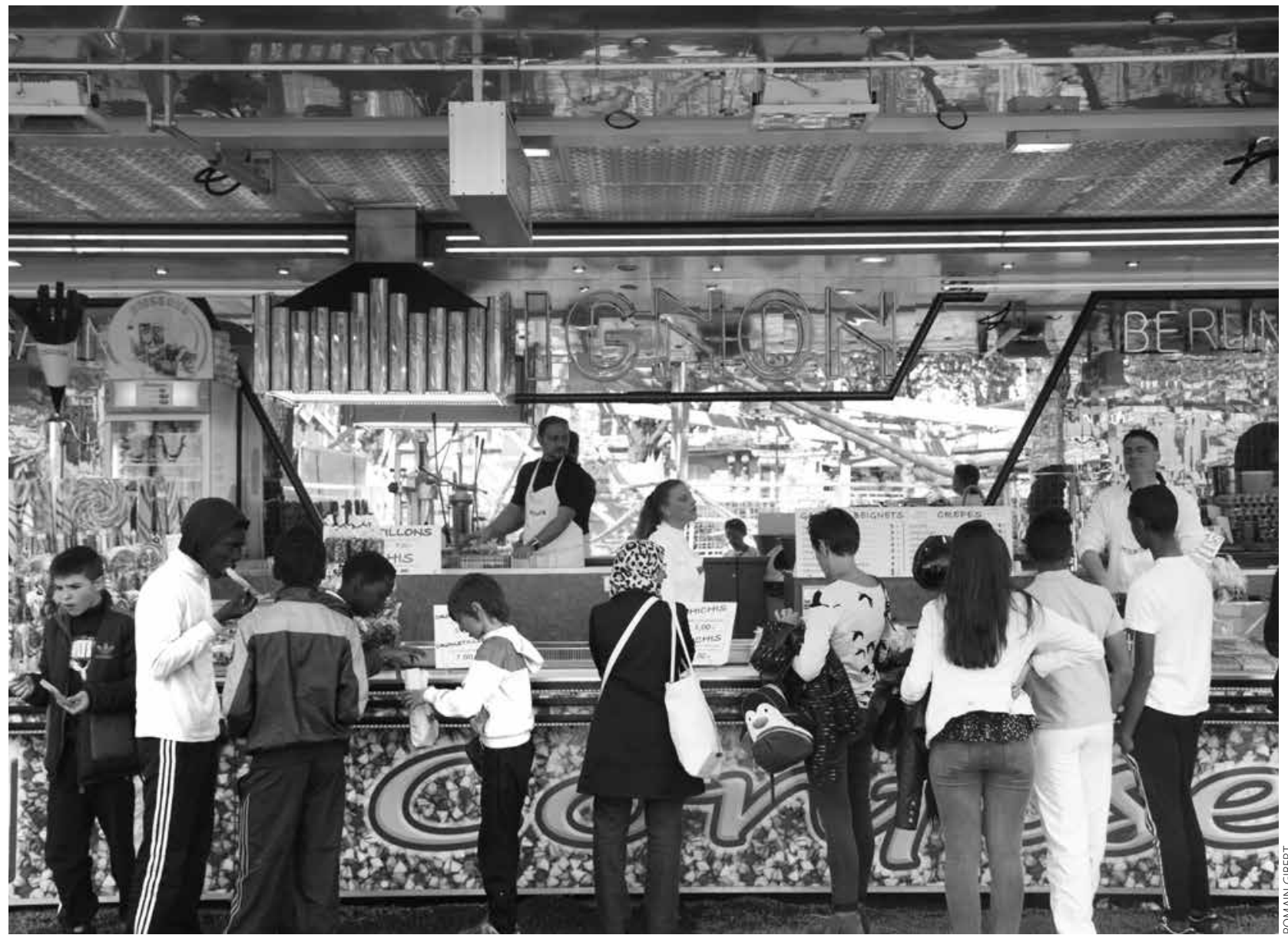

\section{Une place symbolique}

Les adolescents que nous avons rencontrés lors de notre enquête connaissent la représentation négative de leur quartier ainsi que leur place en bas de la hiérarchie sociale, ce que constatait aussi S. Beaud dans la cité HLM de Sochaux-Montbéliard (Amrani et Beaud, 2004). Ils perçoivent l'assignation sociospatiale dévalorisante qu'ils subissent en se comparant à leurs camarades de collège issus d'autre quartier ou commune : «Eux, c'est pas pour dire, mais c'est plutôt des richoux; ils habitent tous en maison. » Cependant, ceux qui s'identifient au quartier opposent à l'image stigmatisée de leur quartier un marquage discursif, symbolique, de " quartier vivant », « animé », où « tout le monde se connaît » et où «y a de l'ambiance ", permettant une identification structurante à leur lieu de résidence. Au moment de l'enquête, des adolescents de Villejean tiraient fierté de la rénovation de leur quartier en montrant avec satisfaction les nouveaux commerces et se réjouissant de la ligne de métro qui les amène très rapidement au centre et au sud de Rennes. Les adolescents de Maurepas qui s'identifient à leur quartier ont cependant une perception plus ambivalente de leur espace de vie : ils valorisent ses relations communautaires et les équipements pour les adolescents, mais souhaitent une rénovation du secteur, en déplorant les problèmes liés à la pauvreté, au chômage, à l'économie parallèle ${ }^{26}$.

Dans les deux quartiers, des adolescents apprennent la partition genrée des espaces, laissent faire, voire reproduisent ce marquage symbolique des lieux. Les garçons ont une réputation d'individu viril à conserver, les filles une réputation de «fille bien » à entretenir, ce qui passe aussi par leur occupation de l'espace public urbain. Une mauvaise réputation peut marquer ceux et celles qui ne respectent pas, de façon réelle ou supposée, leur place, les lieux et les comportements perçus comme convenables pour les adolescents garçons et pour les adolescentes : "Samia est une allumeuse » dit Elvis ; «Gwenn, c'est une racaille » affirme Stéphanie. Ces aspects ont été également constatés dans les quartiers populaires toulousains (Kebabza, 2004) et dans les banlieues parisiennes (Clair, 2008). Dans nos deux quartiers d'enquête, la plupart des adolescent.e.s de quatorze ou quinze ans tentent d'éviter la stigmatisation et une mise à l'écart en se conformant extérieurement à la place qui leur est affectée.

26. Un projet de rénovation urbaine démarre en lien avec l'arrivée du métro à proximité du quartier. 
La notion de marquage, comme expression sociospatiale de différenciation, permet de rendre compte de la constitution d'une place dans l'espace public, ici celle des adolescents et des adolescentes résidant en zone urbaine sensible. Éclairer les marquages des politiques publiques, des professionnels et des habitants, qui attribuent une place aux adolescent.e.s, et les marquages dont ils sont les acteurs, permet de montrer que la place des adolescent.e.s dans les espaces publics découle des rapports sociospatiaux, mais aussi de leurs actes de différenciation et d'expression. Autrement dit, ils/elles ne subissent pas passivement la définition sociale de ce qu'est ou devrait être leur place, mais se l'approprient, l'élargissent, la modifient par leurs investissements signifiants de l'espace. Le marquage dont les adolescents sont sujets et celui dont ils sont objets dans le quartier d'enquête peuvent être analysés comme modalité concrète de la négociation identitaire entre « identité pour soi » et « identité pour autrui » (Dubar,
1991). Ainsi, dans les deux Zus investiguées ${ }^{27}$, les politiques publiques envisagent une place a priori mixte aux adolescents et adolescentes du quartier. Toutefois, sous l'effet des normes habitantes d'appropriation masculine des espaces publics, normes non contrées par les professionnels, les places des adolescent.e.s se différencient selon leur genre et selon leur rapport au quartier. En occupant l'espace commun de la Zus, en y apposant leurs propres marques, des « adolescents du quartier »(Oppenchaim, 2011) s'inscrivent, en la réinterprétant, dans la place sociale du jeune homme qui prévaut dans leur quartier. En délaissant les équipements, en s'appropriant d'autres lieux publics peu visibles ou hors quartier, des adolescentes filles (proches " des filles de bonnes familles » et des «flâneuses » d'Oppenchaim) se

27. Il peut en être différemment dans d'autres Zus.

\section{Références bibliographiques}

Amrani Y., Beaud S., (2004). Pays de malheur! Un jeune de cité écrit à un sociologue, suivi de Des lecteurs nous ont écrit, Paris, La Découverte.

Augustin J.-P., (2001), «Les jeunes entre équipements et espaces publics », Agora débats jeunesses, n²4, pp. 9-18.

Beaud S., (2002), $80 \%$ au bac... et après ? Les enfants de la démocratisation scolaire, Paris, La Découverte.

Beaud S., Pialloux M., (2005), Violences urbaines, violence sociale. Genèse des nouvelles classes dangereuses, Paris, Hachette.

Bréviglieri M., (2007), « L'arc expérientiel de l'adolescence : esquive, combine, embrouille, carapace et étincelle... », Éducation et Sociétés, 1, n 19, pp. 99-113.

Bréviglieri M., Cicchelli V., (2007), Adolescences méditerranéennes. L'espace public à petits pas, Paris, Injep/L'Harmattan (Débats Jeunesses).

Brunet R., Ferras R., Théry H., (1992), Les mots de la géographie. Dictionnaire critique, Paris, Reclus/La Documentation française.

Bruno P., (2000), Existe-t-il une culture adolescente?, Paris, In Press Éditions.

Callu E., Jurmand J.-P., Vulbeau A. (dir), (2005), La place des jeunes dans la cité, t. 2, Espaces de rue, espaces de parole, Paris, L'Harmattan.
Choquet M., (2005), La santé des 14-20 ans, Paris, Inserm.

Clair l., (2008), Les jeunes et l'amour dans les cités, Paris, Armand Colin.

Danic I., Delalande J., Rayou P., (2006), Enquêter auprès d'enfants et dejeunes, Rennes, Pur.

Danic l., (2011), « Investissements ordinaires de l'espace d'un quartier périphérique par les adolescents : une conflictualité sans mobilisation », in Bonny Y., Le Caro Y., Keerle R., et David O., Legrand É., Loncle P., Muniglia V., Rothé C., (2009), « Monographie 1 : état des lieuxà partir des données existantes. Rapport de synthèse de littérature Ille-et-Vilaine », in Les jeunes vulnérables en IIleet-Vilaine. Perceptions et prises en charge dans les politiques sociales et de santélocales, projet de recherche ANR Juvenil, coordonné par I'EHESP, mai, non publié.

David O., Loncle P., (2010), « Monographie 2 : état des lieux de la phase "élus et décideurs"», in Lesjeunes vulnérables en Ille-et-Vilaine. Perceptions et prises en charge dans les politiques sociales et de santélocales, projet de recherche ANR Juvenil, coordonné par l'EHESP, mars, non publié.

Dubar C., (1991), La socialisation. Construction des identités sociales et professionnelles, Paris, Armand Colin.

Dubet F., Martucelli D., (1996), À lécole. Sociologie de l'expérience scolaire, Paris, Seuil.

Faure S., (2008), « Les espaces de socialisation. Garçons et filles d'un quartier HLM», La Revue. Les Rencontres de Bellepierre, $n^{\circ} 3$, [en ligne] www.Irdb.fr, (mis en ligne le 12 sept. 2008)
Kebabza H., (2004), « Logiques de genre dans des quartiers impopulaires », Hommes et migrations, $n^{\circ} 1248$, mars-avril.

Kokoreff M., (1993), « L'espace des jeunes: territoires, identités et mobilité ", Annales de la recherche urbaine, $n^{\circ} 59-60$.

Lagrange H., Oberti M., (2006), Émeutes urbaines et protestations. Une singularité française, Paris, Presses de Sciences Po.

Le Goaziou V., Mucchielli L. (dir.), (2006), Quand les banlieues brûlent. .. Retour sur les émeutes de novembre 2005, Paris, La Découverte.

Loncle P. (dir), (2007), « Jeunes et politiques : amours et désamours », in Les jeunes. Questions de société, questions de politique, Paris, La Documentation française.

Mauger G., (2006), Lémeute de novembre 2005. Une révolte protopolitique, Paris, Éd du Croquant.

Ménard F., Zucker E. (coord.), (2007), « Circulations juvéniles et usages adolescents de l'espace », Sociétés et jeunesses en difficulté, $n^{\circ} 4$, automne, [en ligne] http://sejed.revues.org/index2223.html, (mis en ligne le 27 mars 2008, consulté le 19 mars 2014).

Octobre S., (2004), Les loisirs culturels des 6-14 ans, Paris, La Documentation française. 
redéfinissent une place physique et simultanément une place sociale. À l'opposé, les jeunes du quartier sans investissement extérieur autre que leur collège, proches des « encadrés » d'Oppenchaim (2011) ou ceux que l'on pourrait nommer les « retirés » lorsque leur retrait découle de leur propre décision, n’ont pas de place dans l'espace public, aux sens physique et citoyen du terme.

Comme en témoignent notamment les disparités salariales et les violences conjugales, les rapports dissymétriques entre hommes et femmes traversent notre société et sont présents dans tous les quartiers urbains ${ }^{28}$ (Singly de, 1993). Pour autant, les statuts, les fonctions et les rôles des hommes et des femmes n'y sont pas définis de la même façon, et cela se traduit par des hiérarchies de genre

28. Comme dans les communes périurbaines ou rurales d'ailleurs. plus ou moins marquées selon les espaces ${ }^{29}$. Ceci ne doit pas conduire à naturaliser ou à stigmatiser les rapports sociaux constatés dans les Zus qui résultent des relations mises en œuvre par certains habitants investis dans le quartier, les uns originaires de pays où les rapports entre hommes et femmes sont souvent inégaux, les autres démunis économiquement et culturellement, et partageant un point de vue hiérarchique entre hommes et femmes (Schwartz, 1990). Ceci ne doit pas conduire non plus à une acceptation résignée de l'appropriation masculine des espaces publics des Zus.

29. Beaucoup des adolescentes de Zus rencontrées apprécient de sortir au centre-ville pour sa norme d'égalité entre hommes et femmes et le sentiment de liberté qu'il leur procure.
Octobre S., Detrez C., Merckle P., Berthomier N., (2010), L'enfance des loisirs. Trajectoires communes et parcours individuels de la fin de l'enfance à la grande adolescence, Paris, La Documentation française

Oppenchaim N., (2011), Mobilité quotidienne, socialisation et ségrégation: une analyse à partir des manières d'habiter des adolescents de zones urbaines sensibles, thèse de doctorat de sociologie, université Paris Est.

Pinçon M., Pinçon-Charlot M., (1989), Dans les beaux quartiers, Paris, Puf.

Pinçon M., Pinçon-Charlot M., (2007), Les Ghettos du Gotha: Comment la bourgeoisie défend ses espaces, Paris, Seuil.

Raibaud Y., (2007), « Genre et loisirs des jeunes », Empan, 1, n65, pp. 67-73.

Ripoll F., (2006), « Réflexions sur les rapports entre marquage et appropriation de l'espace », in Bulot T. et Veschambre V. (dir), Mots, traces, marques: dimension spatiale et linguistique de la mémoire urbaine, Paris, L'Harmattan, pp. 15-36.

Rubi S., (2005), «Les crapuleuses », ces adolescentes déviantes, Paris, Puf.

Schwartz O., (1990), Le monde privé des ouvriers, Paris, Puf.

Singly de F., (1993), « Les habits neufs de la domination masculine», Esprit, novembre, pp. 54-64.
Singly de F., (2006), Les adonaissants, Paris, Armand Colin.

Tersigni S., Bonny Y., (2010), « Marques, marqueurs et traces : le marquage des différences dans l'espace », ESO Travaux et documents, $n^{\circ} 30$, décembre.

Veschambre V., (2006), « Patrimonialisation, démolition, mise en mémoire : processus de marquage et d'appropriation symbolique de l'espace », mémoire d'habilitation à diriger des recherches.

Welzer-Lang D., (2002), « Virilité et virilisme dans les quartiers populaires en France, rapports de sexe, rapports de genre », VElenjeux, $n^{\circ} 128$, mars.

Zaffran J., (2000), Les collégiens, l'école et le temps libre, Paris, Syros.

Zaffran J., (2005), « Monter en âge, descendre au centre-ville», in Montulet B. et al. (éds), Mobilités et temporalités, Bruxelles, Publication des Facultés universitaires Saint-Louis.

Zegnani S., (2013), Dans le monde des cités, de la galère à la mosquée, Rennes, Pur.

\section{Biographie}

ISABELLE DANIC est maître de conférences en sociologie à l'université Rennes 2 et membre du laboratoire pluridisciplinaire Espaces et Sociétés (Eso) (unité mixte de recherche CNRS 6590). Ses recherches portent sur la socialisation, l'enfance, I'adolescence, la jeunesse, dans les sphères familiale, scolaire et du temps libre. Parmi ses publications, on peut citer en lien avec l'espace urbain : avec O. David et S. Depeau (dir), Enfants et jeunes dans les espaces du quotidien, Rennes, Pur, 2010 ; «Investissements ordinaires de l'espace d'un quartier périphérique par les adolescents : une conflictualité sans mobilisation », in Bonny Y., Le Caro Y., Keerle R., et Ollitrault S. (dir), Espaces de vie, Espaces-enjeux:entre investissements ordinaires et mobilisations politiques, Rennes, Pur, 2011 ; et « Le rapport à la culture des filles d'un quartier périphérique » in Octobre S. et Sirota R. (dir), Enfance et cultures: regards des sciences humaines et sociales, actes du colloque, ministère de la Culture, [en ligne] www.enfanceetcultures.culture.gouv.fr, 2010.

isabelle.danic@uhb.fi 